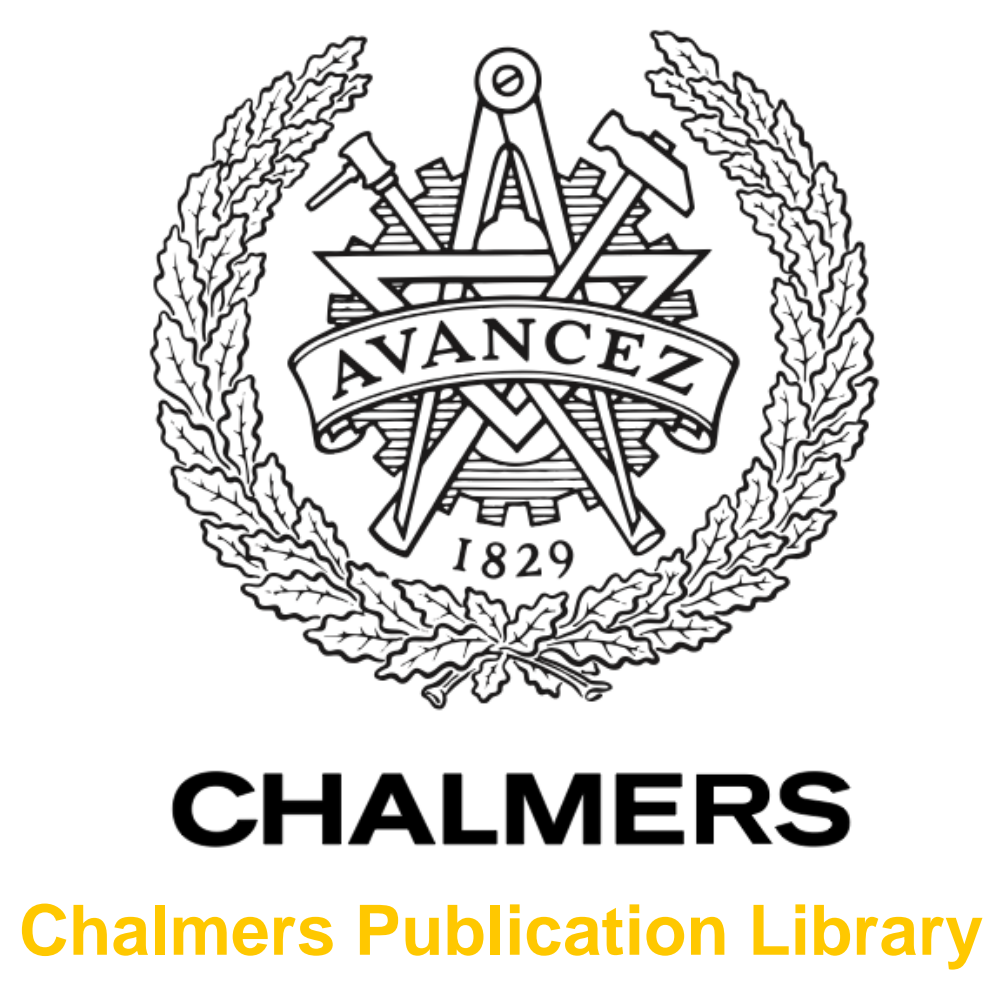

\title{
Performance evaluation of far-field patterns for radio astronomy applications through the Jacobi-Bessel series
}

This document has been downloaded from Chalmers Publication Library (CPL). It is the author's version of a work that was accepted for publication in:

2012 International Conference on Electromagnetics in Advanced Applications (ICEAA), Cape Town, WP, South Africa, 2-8 Sept 2012

Citation for the published paper:

Young, A. ; Maaskant, R. ; Ivashina, M. (2012) "Performance evaluation of far-field patterns for radio astronomy applications through the Jacobi-Bessel series". 2012 International Conference on Electromagnetics in Advanced Applications (ICEAA), Cape Town, WP, South Africa, 2-8 Sept 2012 pp. 884-887.

http://dx.doi.org/10.1109/ICEAA.2012.6328760

Downloaded from: http://publications.lib.chalmers.se/publication/163392

Notice: Changes introduced as a result of publishing processes such as copy-editing and formatting may not be reflected in this document. For a definitive version of this work, please refer to the published source. Please note that access to the published version might require a subscription. 


\title{
Performance Evaluation of Far Field Patterns for Radio Astronomy Applications through the Use of the Jacobi-Bessel Series
}

\author{
André Young* Rob Maaskant ${ }^{\dagger} \quad$ Marianna V. Ivashina ${ }^{\ddagger} \quad$ David B. Davidson ${ }^{\S}$
}

\begin{abstract}
The Jacobi-Bessel (JB) series representation for modeling reflector antenna patterns is proposed to be used as a reference tool to assess and improve the pattern shape of multi-beam wide-scan radio telescopes. After expanding each beam in JB functions, the number of dominant JB functions per beam not only serves as an indication of the beam calibratability (beam smoothness), but is a measure for the uniformity of the scanned patterns as well (inter-beam comparison). It is demonstrated that the first term in the JB series can be used to define directional constraints in a linear constrained minimum variance beamformer, so that each realized scanned beam pattern conforms to this first term. As a result, multiple beams cover a wide field of view in an almost undistorted manner.
\end{abstract}

\section{INTRODUCTION}

With the advent of modern phased array feeds (PAFs) using digital beamforming to illuminate reflector antennas, a single radio telescope can provide multiple closely overlapping beams to increase the size of the Field-of-View (FoV). While this implies that a larger area of the sky can be imaged instantaneously without having to rotate the instrument mechanically, its calibration - which requires a fast characterization of the beam patterns over a large area - becomes a challenging task. In fact, the reflector antenna pattern is a rapidly varying function (so many measurement points are needed to predict it accurately) and should be measured for each of the multiple beams within the FoV.

One solution is to beamform the antenna patterns such that these conform to smooth beams which can be modeled accurately through a few analytical functions. The corresponding function parameters can be determined by measuring the beam in a few angular directions only. Note that,

\footnotetext{
*Department of Electrical and Electronic Engineering, Stellenbosch University, Stellenbosch, South Africa, Email: ayoung@sun.ac.za

$\dagger$ Department of Signals and Systems, Chalmers University of Technology, Gothenburg, Sweden, Email: maaskant@chalmers.se

${ }^{\ddagger}$ Department of Earth and Space Sciences, Chalmers University of Technology, Gothenburg, Sweden, Email: ivashina@chalmers.se

$\S$ Department of Electrical and Electronic Engineering, Stellenbosch University, Stellenbosch, South Africa, Email: davidson@sun.ac.za
}

for reflector antennas, it is natural to expand the beams in terms of the Jacobi-Bessel (JB) series [1], so that the first JB term can be used as a reference beam. Beam shaping for PAF-fed reflector antennas has been proposed and realized in [2] through the use of a linear constrained minimum variance (LCMV) beamformer. While in [2] the directional constraints are chosen to minimize the sensitivity ripple over the FoV, in this work, the most dominant JB function (first term) is used as a physics-based reference beam for determining the directional constraints. The advantage of the latter beamforming approach is that each beam conforms naturally and therefore relatively easily to the JB reference beam over a larger angular region and FoV. Furthermore, the magnitude of the higherorder terms in the JB expansion provides us with a measure for the beam calibratability; for instance, if all beams within the FoV can be assumed to have a strongly dominant first JB term, the beam calibration involves measuring only the first JB function parameters, possibly for one beam only.

\section{ANTENNA PATTERN MODEL}

Assuming that a far field pattern $\mathbf{E}(\theta, \phi)$ is defined in a reference frame in which its maximum is at $\theta=$ 0 , then over a small angular region the pattern can be approximated by $\tilde{\mathbf{E}}(\theta, \phi)$, which is a simplified form of the series solution presented in [1],

$$
\begin{aligned}
\tilde{\mathbf{E}}(\theta, \phi)=2 \pi & a^{2} \sum_{n=0}^{N} \sum_{m=0}^{M} j^{n}\left(\mathbf{C}_{n, m} \cos n \phi\right. \\
& \left.+\mathbf{D}_{n, m} \sin n \phi\right) \cdot \sqrt{2 q} \frac{J_{q}(k a \theta)}{k a \theta} .
\end{aligned}
$$

Here $q=n+2 m+1, J_{q}(x)$ is the $q$ th order Bessel function of the first kind and $k$ is the free space wavenumber. The parameter $a$ is an effective aperture radius which is related to the physical aperture radius $a_{\mathrm{ph}}$ through the scaling factor $s$ and $a=s a_{\mathrm{ph}}$. The factor $s$ controls the angular width of the analytical beam functions and is typically close to 1 . The coefficients $\mathbf{C}_{n, m}, \mathbf{D}_{n, m}$ are the vector-valued (two polarization components) unknown coefficients that need to be solved in order 
to satisfy the condition $\tilde{\mathbf{E}} \approx \mathbf{E}$ within a certain angular region $0 \leq \theta \leq \theta_{W}$. These coefficients can be determined through the approximate closed-form solution

$$
\begin{aligned}
\left\{\begin{array}{l}
\mathbf{C}_{n m} \\
\mathbf{D}_{n m}
\end{array}\right\}=\frac{\sqrt{n q}}{2 \pi a^{2} j^{n}} \int_{0}^{\theta_{W}} J_{q}(k a \theta) \\
\cdot \frac{\epsilon_{n}}{2 \pi} \int_{0}^{2 \pi}\left\{\begin{array}{c}
\cos n \phi \\
\sin n \phi
\end{array}\right\} \mathbf{E}(\theta, \phi) d \phi d \theta
\end{aligned}
$$

where $\epsilon_{n}=2$ for $n>0, \epsilon_{n}=1$ for $\mathbf{C}_{0, m}$, and all $\mathbf{D}_{0, m}$-coefficients are identically zero. Herein only the co-polarized field component is considered.

The first term in (1) is proportional to the jinc function, $\operatorname{jinc}(k a \theta)=J_{1}(k a \theta) / k a \theta$, which is approximately equal to the pattern created by a circular aperture of radius $a=a_{\mathrm{ph}}$ with a uniform aperture field distribution [3]. Patterns that deviate from this reference pattern, e.g. the patterns due to a non-uniform aperture field distribution with some phase gradient (that is the case for off-axis beams) will require more terms in the approximation $\tilde{\mathbf{E}}$ to achieve a certain level of accuracy. The number of required terms then provides a measure of how much a given pattern deviates from the reference pattern.

\section{BEAMFORMING STRATEGY}

An LCMV beamformer that aims to produce the beam patterns conforming to the first term in (1) while maximizing the signal-to-noise ratio (SNR) in the direction of observation is implemented. The weights applied to the PAF elements are calculated according to $[4]$

$$
\mathbf{w}_{\mathrm{LCMV}}=\mathbf{g}^{H}\left[\mathbf{G}^{H} \mathbf{C}^{-1} \mathbf{G}\right]^{-1} \mathbf{G}^{H} \mathbf{C}^{-1}
$$

in which $\mathbf{x}^{H}$ means the complex conjugate transpose of $\mathbf{x}, \mathbf{C}$ is the noise covariance matrix, $\mathbf{g}$ is the constraints vector, and $\mathbf{G}$ is the directional constraint matrix. Directional constraints are enforced at seven points for each pattern created by the LCMV beamformer: one constraint is at the center of the main beam (the desired scan direction); the other six constraints are spaced circularly symmetric around and $0.25^{\circ}$ off the beam center. The values for the vector $\mathbf{g}$ are calculated by evaluating the first term in (1) (cosine term with $n, m=0$ ) at the $(\theta, \phi)$ coordinates where the constraints are applied, in a reference frame where $\theta=0$ corresponds to the desired scan direction. To compensate for beam widening with scan the scaling $s=0.85$ was used.

For comparison, the standard maximum SNR beamformer [5] (without directional constraints) is also applied for which the weights are equal to

$$
\mathbf{w}_{\text {MaxSNR }}=\mathbf{C}^{-1} \mathbf{v}
$$

and where $\mathbf{v}$ is the signal response vector of the array due to a plane wave incident from the direction of interest.

For the purposes of this study the antenna system is assumed to be noise-less which means $\mathbf{C}$ is proportional to the identity matrix. For the above beamformers this implies that maximizing SNR is equivalent to maximizing directivity.

\section{NUMERICAL RESULTS}

To investigate the proposed beamforming solution for addressing the problem of calibration complexity, we have tested it for a PAF system similar to the one that is currently being built at ASTRON [6]. For this purpose, we have used the PAF numerical model and simulation software tool in [2] and [7], respectively, to compute the embedded element patterns of the PAF-reflector system. From these embedded patterns the compound beams were formed according to the beamformers defined in (3) and (4). The PAF under study consists of 121 tapered slot antenna (TSA) elements, and the reflector has a diameter of $25 \mathrm{~m}$ and focal-length-to-diameter ratio $(F / D)$ of 0.38 . For comparison, a conical horn feed is also included in the analysis for the evaluation of the beam calibratability.

Fig. 1 shows the on-axis pattern for the hornfed reflector antenna, as well as a number of approximate patterns calculated using (1) and $s=1$. The first term $C_{0,0}$ provides an accurate approximation to the pattern only over a very narrow region (within about 0.6 of the HPBW). The accuracy of the approximate main beam is greatly improved by including two more terms, $C_{0,1}$ and $C_{0,2}$, to account for the relatively wide beamwidth resulting from the edge taper of the reflector illumination. Although the main lobe of the pattern is modeled accurately with so few terms, a much larger number of terms is required to also model the first few sidelobes accurately; for instance, at least seven and ten terms are required to model the first and second side lobes, respectively.

Fig. 2 shows the simulated on-axis beam pattern for the array-fed reflector antenna calculated for the maximum SNR beamformer according to (4), as well as a number of approximate patterns computed through (1) with $s=1$. For the beamformed array feed, a number of degrees of freedom (DoFs) for the excitation weight vector are available for optimizing the reflector aperture field distribution, in this case to create a near uniform field distribution. Consequently, a higher illumination efficiency 
is attainable for the array-fed reflector than for the horn-fed reflector. As a result, the array-fed reflector with the maximum SNR beamformer produces a pattern which is modeled fairly accurately with the first term, $C_{0,0}$, of (1), especially over the main beam, as shown in Fig. 2. As before, a large number of terms are required to obtain an accurate approximation of the first few side lobes.

The above analysis has also been carried out for a number of scanned patterns, for both the hornfed and array-fed reflectors. For every scan the obtained pattern was compared to the on-axis pattern obtained with the same feed, and the average relative difference between these patterns was computed. Fig. 3 shows these results for the horn-fed reflector, and the array-fed reflector for the maximum SNR beamformer as well as for the LCMV beamformer. For the array-fed reflector, scanning was achieved through adjusting the beamformer weights, as well as by displacing the feed laterally in order to avoid array truncation effects at large scan angles. The results show that both the horn feed and array feed with the maximum SNR beamformer realize patterns that vary rapidly with scan angle (more than $10 \%$ over 5 beamwidths), while the patterns realized with the LCMV beamformer array are much more uniform (less than $3 \%$ over 5 beamwidths).

The number of terms in (1) required to model each of these scanned patterns with sufficient accuracy (less than $10 \%$ average relative error over the $10 \mathrm{~dB}$ beam) is shown in Fig. 4. For the horn-fed and maximum SNR beamforming array-fed reflectors the scaling $s=1$ was used, whereas for the array-fed reflector with LCMV beamforming the scaling $s=0.85$ was used. As expected, the pat-

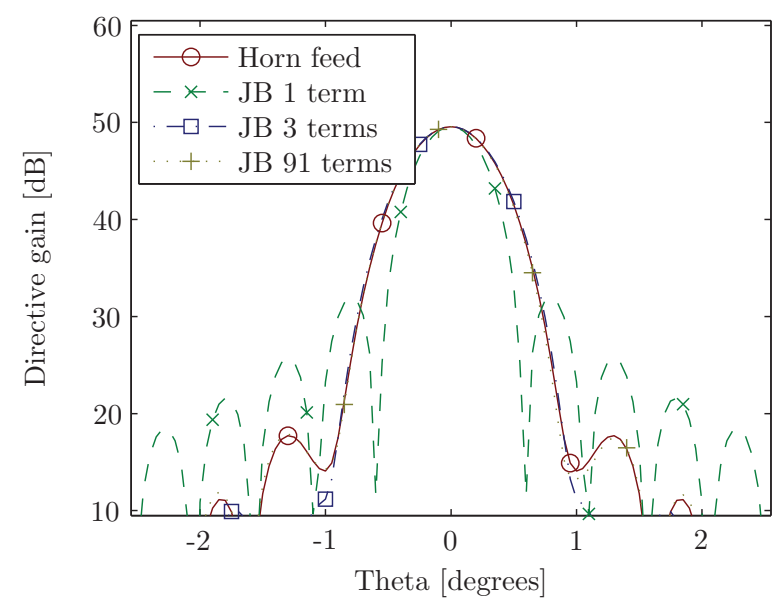

Figure 1: On-axis horn-fed reflector antenna pattern and pattern models using 1, 3, and 91 terms.

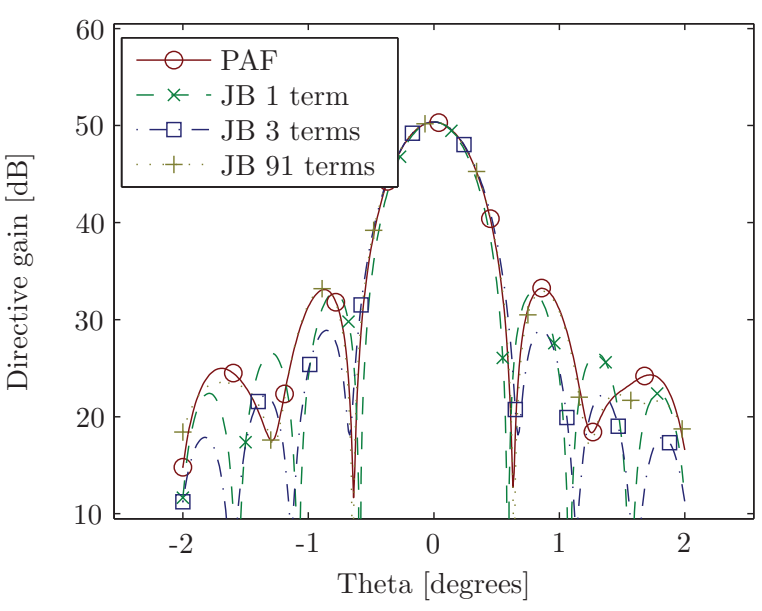

Figure 2: On-axis array-fed reflector antenna pattern and pattern models using 1, 3, and 91 terms.

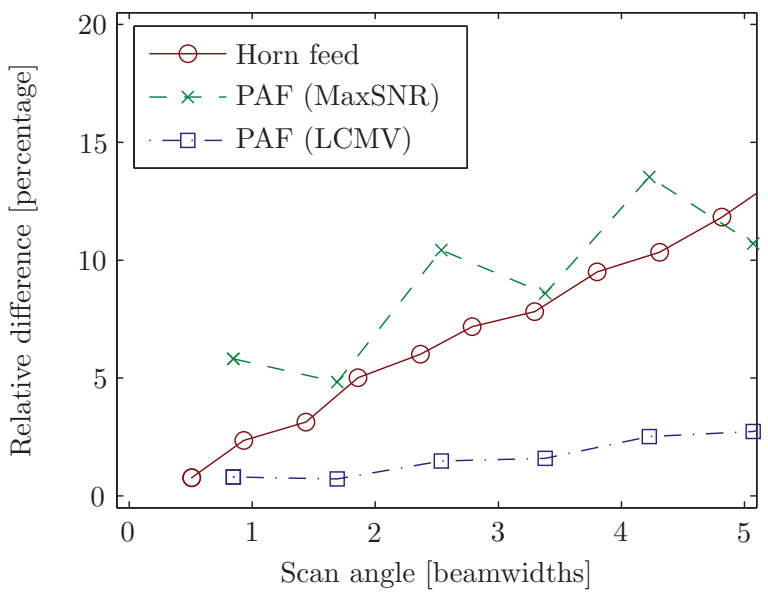

Figure 3: Average relative difference between onaxis and scanned patterns for different feeds (computed over the $10 \mathrm{~dB}$ beam of the on-axis pattern).

terns obtained with the LCMV beamformer array require the least number of terms; in fact, a single term models the scanned beam patterns with sufficient accuracy over the entire FoV. Also, for the maximum SNR beamformer array, a single term is adequate for modeling the on-axis pattern, but more terms are required to maintain the same level of accuracy for scanned patterns. For the hornfed reflector two terms are required - even for the on-axis pattern - while this number increases with increasing scan angle.

Since some of the DoFs available to the LCMV beamformed array are used to satisfy a number of directional constraints, less DoFs are available to maximize the SNR. It is therefore expected that the directivities of the patterns obtained with the 


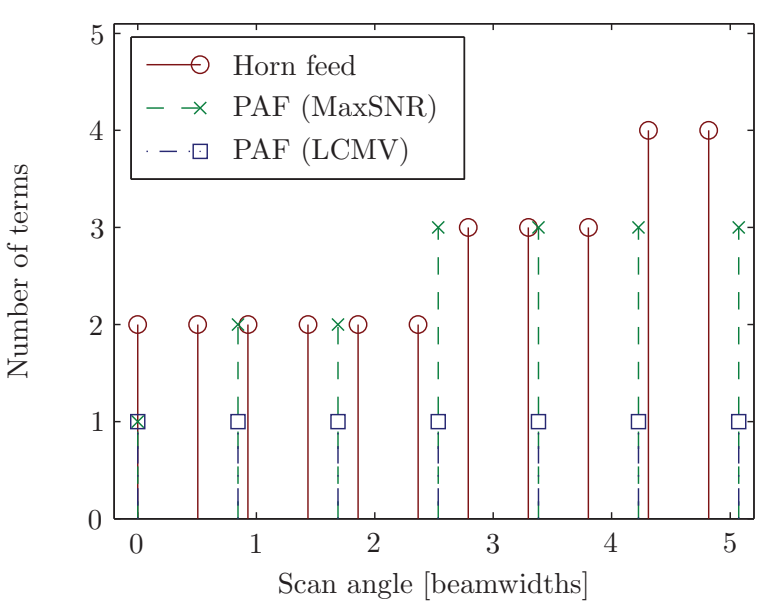

Figure 4: Number of terms in the pattern model to achieve $10 \%$ accuracy over the $10 \mathrm{~dB}$ beam of the single reflector antenna.

maximized SNR beamformed array are higher than those obtained with the LCMV beamformed array. Within a range of scan angles of \pm 3 beamwidths (implying at least 7 multiple beams in one plane), the directivity for the LCMV beamformer is within $0.3 \mathrm{~dB}(7 \%)$ below that for the maximum SNR beamformer. This deficit increases slightly at larger scan angles, but remains less than $0.5 \mathrm{~dB}$ (10\%) for a scan up to \pm 5 beamwidths, providing a very large number of possible scanned beams and greatly increased size of the FoV. This result is very promising, since other LCMV beamformers proposed thus far, including those developed in [2] and by the DRAO [8], have reported relative sensitivity reductions in the order of $20-25 \%$. However, it should be noted that the present beamforming strategy is yet to be evaluated in the presence of correlated noise. This is left for future studies.

\section{CONCLUSIONS}

The Jacobi-Bessel (JB) functions have been used to accurately expand the reflector antenna beam patterns over a wide FoV. Both the number and magnitude of the resulting JB function parameters have been used as a reference tool to evaluate the calibration complexity of horn- and array-fed reflector antennas. The first JB function has been used as the smooth reference beam for defining the pattern constraints in an LCMV beamformer. The realized scanned beam patterns were shown to conform to this reference beam over a wide FoV of up to \pm 5 beamwidths, which is a significant improvement as compared to the calibratability of the horn and maximum SNR beamformed array feeds. Further- more, the patterns obtained with the LCMV beamformed array feed were more uniform over the FoV. However, the LCMV beamformer compromised the sensitivity, as opposed to the maximum SNR beamformer, but this loss in sensitivity was less than $10 \%$ for wide scanning up to 5 beamwidths off-axis.

\section{Acknowledgment}

The authors would like to thank O. Iupikov for making the GRASP toolbox available for this work. A. Young and D. B. Davidson acknowledge the support of the South African Research Chairs Initiative of the Department of Science and Technology (DST) and National Research Foundation (NRF). Any opinion, findings and conclusions or recommendations expressed in this material are those of the authors and therefore the NRF and DST do not accept any liability with regard thereto.

\section{References}

[1] Y. Rahmat-Samii and V. Galindo-Israel, "Shaped reflector antenna analysis using the Jacobi-Bessel series," IEEE Trans. Antennas Propag., vol. 28, no. 4, pp. 425-435, Jul. 1980.

[2] O. A. Iupikov, M. V. Ivashina, and O. M. Smirnov, "Reducing the complexity of the beam calibration models of phased-array radio telescopes," in Proc. EuCAP, Apr. 2011, pp. 930933.

[3] W. L. Stutzman and G. A. Thiele, Antenna Theory and Design, 2nd ed. New York, N.Y.: Wiley, 1998.

[4] H. L. van Trees, Optimum Array Processing. New York, N.Y.: Wiley, 2002.

[5] D. K. Cheng and F. I. Tseng, "Maximisation of directive gain for circular and elliptical arrays," Proc. Inst. Elec. Eng., vol. 114, no. 5, pp. 589594, May 1967.

[6] M. A. W. Verheijen, T. A. Oosterloo, W. A. van Cappellen, L. Bakker, M. V. Ivashina, and J. M. van der Hulst, "APERTIF, a focal plane array for the WSRT," 2008. [Online]. Available: http://arxiv.org/abs/0806.0234v1

[7] M. V. Ivashina, O. A. Iupikov, R. Maaskant, W. A. van Cappellen, and T. Oosterloo, "An Optimal Beamforming Strategy for WideField Surveys With Phased-Array-Fed Reflector Antennas," IEEE Trans. Antennas Propag., vol. 59, no. 6, pp. 1864-1875, Jun. 2011.

[8] A. Willis, 2011, private communication. 